Proceedings of the Edinburgh Mathematical Society (2004) 47, 325-337 (C)

DOI:10.1017/S0013091503000051 Printed in the United Kingdom

\title{
ON UPPER-MODULAR SUBALGEBRAS OF A LIE ALGEBRA
}

\author{
KEVIN BOWMAN ${ }^{1}$, DAVID A. TOWERS ${ }^{2}$ AND VICENTE R. VAREA ${ }^{3}$ \\ ${ }^{1}$ Department of Physics, Astronomy and Mathematics, \\ University of Central Lancashire, Preston PR1 2HE, UK \\ ${ }^{2}$ Department of Mathematics, Lancaster University, \\ Lancaster LA1 4YF, UK (d.towers@lancaster.ac.uk) \\ ${ }^{3}$ Department of Mathematics, University of Zaragoza, \\ Zaragoza, 50009 Spain
}

(Received 30 January 2003)

\begin{abstract}
This paper is a further contribution to the extensive study by a number of authors of the subalgebra lattice of a Lie algebra. We give some necessary and some sufficient conditions for a subalgebra to be upper modular. For algebraically closed fields of any characteristic these enable us to determine the structure of Lie algebras having abelian upper-modular subalgebras which are not ideals. We then study the structure of solvable Lie algebras having an abelian upper-modular subalgebra which is not an ideal and which has trivial intersection with the derived algebra; in particular, the structure is determined for algebras over the real field. Next we classify non-solvable Lie algebras over fields of characteristic zero having an upper-modular atom which is not an ideal. Finally, it is shown that every Lie algebra over a field of characteristic different from two and three in which every atom is upper modular is either quasi-abelian or a $\mu$-algebra.
\end{abstract}

Keywords: Lie algebra; subalgebra lattice; upper modular

2000 Mathematics subject classification: Primary 17B05; 17B50; 17B30; 17B20

\section{Introduction}

This paper is a further contribution to the extensive study by a number of authors of the subalgebra lattice of a Lie algebra. A subalgebra $U$ of a Lie algebra $L$ is called upper modular in $L$ if, whenever $B$ is a subalgebra of $L$ which covers $U \cap B$ (that is, such that $U \cap B$ is a maximal subalgebra of $B$ ), then $\langle U, B\rangle$ covers $U$. (Here, $\langle U, B\rangle$ denotes the subalgebra of $L$ generated by $U$ and $B$.) This concept was introduced in [10] and studied further in $[\mathbf{2}]$. Our objective here is to gain deeper insight into the structure of Lie algebras $L$ containing an upper-modular subalgebra with certain extra properties, and to the position of such a subalgebra within $L$.

In $\S 2$ we assemble some results relating upper-modular subalgebras to ideals in the algebra. We also give a straightforward characterization of upper-modular subalgebras of supersolvable Lie algebras. In $\S 3$ we give some sufficient conditions and some necessary conditions for a subalgebra to be upper modular. For algebraically closed fields of any 
characteristic, we determine the structure of Lie algebras having abelian upper-modular subalgebras which are not ideals. Section 4 is concerned with studying the structure of solvable Lie algebras $L$ having an abelian upper-modular subalgebra $U$ which is not an ideal of $L$ and such that $U \cap L^{2}=0$. We determine this structure when the ground field is the real numbers; for more general fields, we need to impose the condition that $L^{2}$ is abelian.

In $\S 5$ we classify non-solvable Lie algebras over fields of characteristic zero having an upper-modular minimal subalgebra which is not an ideal. Such algebras exist if and only if $\sqrt{F} \not \subset F$, where $F$ is the underlying field. The final section is devoted to Lie algebras $L$ in which every minimal subalgebra is upper modular in $L$. We obtain that, over a field of characteristic different from 2,3 , such an algebra is either quasi-abelian or a $\mu$-algebra.

Throughout, $L$ will denote a finite-dimensional Lie algebra over a field $F$. There will be no assumptions on $F$ other than those specified in individual results. The symbol ' $\oplus$ ' will denote a vector space direct sum. We will use the notation $A \subseteq B$ to mean that $A$ is a subset of $B$, whereas $A \subset B$ indicates that $A$ is a proper subset of $B$. Standard results in Lie algebras are taken from $[\mathbf{6}]$.

\section{Preliminaries}

If $U$ is a subalgebra of $L$, then the normalizer of $U$ in $L$ is the set

$$
N_{L}(U)=\{x \in L:[x, U] \subseteq U\} .
$$

First we give two results that have been proved elsewhere, but which will be used extensively later.

Lemma 2.1. Let $U$ be upper modular in $L$. Then $N_{L}(U)=U$ or $L$.

Proof. This is Lemma 1.5 of $[\mathbf{1 0}]$.

Theorem 2.2. Let $U$ be upper modular in $L$ and let $I$ be an ideal of $L$ such that $U \subseteq I \neq L$. Then $U$ is an ideal of $L$.

Proof. This is Lemma 2.2 of $[\mathbf{9}]$.

The centre of $L, Z(L)$, is defined by $Z(L)=\{x \in L:[x, L]=0\}$. If $U$ is a subalgebra of $L$, the core of $U, U_{L}$, is the largest ideal of $L$ contained in $U$. We now have the following corollary to the above result.

Corollary 2.3. Let $U$ be an upper-modular subalgebra of $L$ which is not an ideal of $L$. Then $Z(L) \subseteq U_{L}$.

Proof. Clearly, $Z(L) \subseteq N_{L}(U)=U$.

For $x \in L$, the Engel subalgebra of $x, E_{L}(x)$, is the Fitting null-component of $L$ relative to ad $x$. With a mild restriction on the field, we can improve the above result when $U$ is an abelian upper-modular subalgebra of $L$. 
Theorem 2.4. Let $L$ be a Lie algebra of dimension $n$ over a field $F$ which has at least $n$ elements and let $U$ be an abelian upper-modular subalgebra of $L$ which is not an ideal of $L$. Then $U_{L}=Z(L)$.

Proof. We have that $U$ is nilpotent and self-normalizing, and so is a Cartan subalgebra of $L$. It follows from Theorem 4.6 of [1] that $U$ is minimal Engel in $L$, and so $U=E_{L}(x)$ for some $x \in U$. Write $L=E_{L}(x) \oplus \bar{E}(x)$, where $\bar{E}(x)$ is the Fitting one-component of $L$ relative to ad $x$. Then $L=U \oplus \bar{E}(x)$ and

$$
\left[L, U_{L}\right]=\left[\bar{E}(x), U_{L}\right] \subseteq U_{L} \cap\left[\bar{E}(x), E_{L}(x)\right] \subseteq U_{L} \cap \bar{E}(x)=0 .
$$

Thus $U_{L} \subseteq Z(L)$. Now combine this with the Corollary 2.3 .

A subalgebra $Q$ of $L$ is called a quasi-ideal of $L$ if $[Q, V] \subseteq Q+V$ for every subspace $V$ of $L$. It is easy to see that quasi-ideals of $L$ are always upper-modular subalgebras of $L$. When $L$ is supersolvable, the upper-modular subalgebras of $L$ are precisely the quasiideals of $L$, as is shown next.

Theorem 2.5. Let $L$ be supersolvable and let $U$ be upper modular in $L$. Then $U$ is a quasi-ideal of $L$.

Proof. Suppose that $x \in L \backslash U$. Then $U \cap F x=0$, which is covered by $F x$. Hence $\langle U, x\rangle$ covers $U$ and so, since $\langle U, x\rangle$ is supersolvable, $\langle U, x\rangle=U+F x$.

\section{General results}

Lemma 3.1. Let $L$ be a Lie algebra and let $U$ be a subalgebra of $L$. Let $A$ be any subspace of $L$ such that $L=U \oplus A$. Then $U$ is upper modular in $L$ if and only if $U$ is maximal in $\langle U, a\rangle$, for every $0 \neq a \in A$.

Proof. The 'only if' part is trivial. To prove the converse, take a subalgebra $S$ of $L$ such that $S \cap U$ is maximal in $S$. Pick $0 \neq s \in S, s \notin U$. We have $S=\langle U \cap S, s\rangle$. We have $\langle U, s\rangle \supseteq\langle U \cap S, s\rangle=S$. This yields $\langle U, S\rangle \supseteq\langle U, s\rangle \supseteq\langle U, S\rangle$, from which $\langle U, S\rangle=\langle U, s\rangle$. Now decompose $s=a+u$, where $a \in A$ and $u \in U$. As $s \notin U$, we see that $a \neq 0$. Also, we have $\langle U, s\rangle=\langle U, a\rangle$. By our hypothesis, $U$ is maximal in $\langle U, a\rangle$. Consequently, $U$ is maximal in $\langle U, S\rangle$. Hence $U$ is upper modular in $L$.

Now, for algebraically closed fields of any characteristic, we are able to determine the structure of Lie algebras having abelian upper-modular subalgebras which are not ideals.

Theorem 3.2. Let $F$ be an algebraically closed field of any characteristic. Let $L$ be a Lie algebra and let $U$ be an abelian subalgebra of $L$ which is not an ideal of $L$. Then $U$ is upper modular in $L$ if and only if $L=A \oplus U$, where either $[A, U]=0$, or $A$ is an abelian ideal of $L$ and every element of $U$ acts as a scalar on $A$. 
Proof. Assume that $U$ is upper modular in $L$. By Lemma 2.1, we have that $U$ is a Cartan subalgebra of $L$. Let $L=L_{1}(U) \oplus U$ be the Fitting decomposition of $L$ relative to $U$. Let $0 \neq a \in L_{1}(U)$. Then $U$ is maximal in $\langle U, a\rangle$ because of its upper modularity. As $L_{1}(U)$ is a $U$-submodule of $L$, so is $L_{1}(U) \cap\langle U, a\rangle$. Since $U$ is abelian, we have that $\left\{\left.(\operatorname{ad} u)\right|_{L_{1}(U) \cap\langle U, a\rangle}: u \in U\right\}$ is a set of simultaneously triangulable linear mappings. So there must exist $0 \neq b \in L_{1}(U) \cap\langle U, a\rangle$ such that $[u, b]=\alpha(u) b$ for every $u \in U$, where $\alpha(u) \in F$. Then we have that $U+F b$ is a subalgebra of $\langle U, a\rangle$ strictly containing $U$. Therefore, $U+F b=\langle U, a\rangle$. This yields $L_{1}(U) \cap\langle U, a\rangle=F b$, and hence $F a=F b$. Consequently, every non-zero element of $L_{1}(U)$ is a common eigenvector of ad $u$ for each $u \in U$. From this it follows that ad $u$ acts as a scalar on $L_{1}(U)$, so that $\left.(\operatorname{ad} u)\right|_{L_{1}(U)}=\alpha(u) 1$, where $\alpha(u) \in F$. Then, by using the Jacobi identity, we see that $\left[L_{1}(U), L_{1}(U)\right]=0$ if $\alpha \neq 0$. This yields that either $U$ acts trivially on $L_{1}(U)$, or $L_{1}(U)$ is an abelian ideal of $L$.

To prove the converse, let $0 \neq a \in A$. By our hypothesis, we have $[u, a]=\alpha(u) a$, where $\alpha(u) \in F$, for every $u \in U$. So $U+F a$ is a subalgebra of $L$. Therefore, $\langle U, a\rangle=U+F a$ and $U$ is maximal in $\langle U, a\rangle$. By Lemma 3.1, it follows that $U$ is upper modular in $L$.

Note that the condition that $F$ is algebraically closed in Theorem 3.2 can be removed whenever the subalgebra $U$ is splitting.

Next, we give sufficient conditions to guarantee that a subalgebra is upper modular.

Lemma 3.3. Let $L=U \oplus A$ be a Lie algebra, where $U$ is a subalgebra of $L$ and $A$ is a $U$-submodule of $L$.

(i) Let $B$ be any irreducible $U$-submodule of $A$. Then $\langle U, B\rangle=\langle U, b\rangle$ for every $0 \neq$ $b \in B$.

(ii) If $\langle U, a\rangle \cap A$ is irreducible as a $U$-submodule, for every $0 \neq a \in A$, then $U$ is upper modular in $L$.

Proof. (i) We have

$$
[U,\langle U, b\rangle \cap A] \subseteq[U, A] \cap[\langle U, b\rangle,\langle U, b\rangle] \subseteq A \cap\langle U, b\rangle,
$$

so that $\langle U, b\rangle \cap A$ is a $U$-submodule of $A$. Since $0 \neq b \in B \cap\langle U, b\rangle$ and $B$ is irreducible, we have $B=B \cap\langle U, b\rangle$. This yields $\langle U, B\rangle \subseteq\langle U, b\rangle \subseteq\langle U, B\rangle$, whence $\langle U, b\rangle=\langle U, B\rangle$, as required.

(ii) By Lemma 3.1, we need only prove that $U$ is maximal in $\langle U, a\rangle$ for every $0 \neq a \in A$. To see this, let $M$ be a subalgebra of $\langle U, a\rangle$ such that $U \subseteq M \subseteq\langle U, a\rangle$. We have $M=(M \cap A) \oplus U$. On the other hand, we see that $M \cap A$ is a $U$-submodule of $A$ and $M \cap A \subseteq\langle U, a\rangle \cap A$. So either $M \cap A=0$, and then $M=U$, or $M \cap A=\langle U, a\rangle \cap A$, and then $M=\langle U, a\rangle$.

Theorem 3.4. Let $L=U \oplus A$ be a Lie algebra over any field $F$, where $U$ is a subalgebra of $L$ and $A$ is a $U$-submodule of $L$. Assume, in addition, that the following hold. 
(i) Each element of $A$ lies in an irreducible $U$-submodule of $L$.

(ii) $B+U$ is a subalgebra of $L$, for every irreducible $U$-submodule $B$ of $A$.

Then $U$ is upper modular in $L$.

Proof. Let $0 \neq a \in A$. By (i), there exists a $U$-submodule $B$ of $A$ containing $a$. By Lemma 3.3 (i), we have $\langle U, a\rangle=\langle U, B\rangle$. Since $U+B$ is a subalgebra, we have $\langle U, B\rangle=$ $U+B$, whence $\langle U, a\rangle \cap A=(U+B) \cap A=B$. It follows from Lemma 3.3 (ii) that $U$ is upper modular in $L$.

Condition (i) of Theorem 3.4 is clearly satisfied whenever $U=F u$ and the minimum polynomial of $\left.(\operatorname{ad} u)\right|_{A}$ is irreducible. Also, it is satisfied in the case where the ground field is infinite and perfect, $U$ is abelian and the minimum polynomial of $\left.(\operatorname{ad} u)\right|_{A}$ is irreducible for every $u \in U$, as is shown by the following.

Proposition 3.5. Let $L=U \oplus A$ be a Lie algebra over an infinite perfect field, where $U$ is an abelian subalgebra of $L, A$ is a $U$-submodule of $L$ and the minimum polynomial of $\left.(\operatorname{ad} u)\right|_{A}$ is irreducible for every $u \in U$. Then each element $a \in A$ lies in an irreducible $U$-submodule of $A$.

Proof. Let $\Omega$ be the algebraic closure of $F$, and put $A_{\Omega}=A \otimes_{F} \Omega$. Let

$$
\sigma: U \rightarrow g l(A): u \mapsto(a \mapsto[a, u])
$$

be the representation corresponding to the $U$-module $A$. Pick $u \in U$ and decompose $A$ into its generalized eigenspaces relative to $\sigma(u)$. Since $[\sigma(u), \sigma(v)]=0$ for every $v \in U$, each generalized eigenspace is a submodule of $A$. Also, since the minimum polynomial of $\sigma(u)$ is irreducible for every $u \in U$, the transformations form a set of commuting semisimple operators. It follows that $A_{\Omega}$ can be decomposed into

$$
\left(A_{\Omega}\right)_{\alpha_{i}}=\left\{a \in A_{\Omega}: \tilde{\sigma}(a)=\alpha_{i}(u) a \forall u \in U\right\},
$$

where $\tilde{\sigma}$ is the representation of $A_{\Omega}$ induced by $\sigma$ and $1 \leqslant i \leqslant r$.

If $r=1$, then $A=\sum_{i=1}^{n} F a_{i}$, where $[u, a]_{i}=\alpha_{u} a_{i}$ for all $u \in U$, and the result is clear.

So suppose that $r>1$. Then, as in Lemma 3.2 of [5], there exists $u_{0} \in U$ such that $\alpha_{i}\left(u_{0}\right) \neq \alpha_{j}\left(u_{0}\right)$ for every $1 \leqslant i \neq j \leqslant r$. Pick $0 \neq a \in A$, and put $C=\sum_{k=0}^{\infty} a\left(\operatorname{ad} u_{0}\right)^{k}$, so that $C$ is a cyclic subspace of $A$ relative to $\sigma\left(u_{0}\right)$. Now $\left(A_{\Omega}\right)_{\alpha_{i}}$ is just the eigenspace of $A_{\Omega}$ corresponding to the eigenvalue $\alpha_{i}\left(u_{0}\right)$ relative to $\tilde{\sigma}\left(u_{0}\right)$ for every $1 \leqslant i \leqslant r$. Thus

$$
C_{\Omega}=\bigoplus_{i=1}^{r} C_{\Omega} \cap\left(A_{\Omega}\right)_{\alpha_{i}} .
$$

Let $c \in C$ and write $c=c_{1}+\cdots+c_{r}$, where $c_{i} \in C_{\Omega} \cap\left(A_{\Omega}\right)_{\alpha_{i}}$. Then, for every $u \in U$,

$$
\sigma(u)(c)=\sum_{i=1}^{r} \tilde{\sigma}(u)\left(c_{i}\right)=\sum_{i=1}^{r} \alpha_{i}(u)\left(c_{i}\right) \in C_{\Omega} \cap A=C,
$$

so $C$ is a $U$-submodule of $A$. It is irreducible as such, as $\sigma\left(u_{0}\right)$ has an irreducible minimum polynomial, and contains $a$. 
Now we give necessary conditions for a subalgebra to be upper modular.

Theorem 3.6. Let $L=U \oplus A$ be a Lie algebra, where $U$ is a subalgebra of $L$ and $A=N \oplus V$ for some non-zero solvable ideal $N$ of $L$ and an irreducible $U$-submodule $V$ of $L$ (possibly zero). Let $0 \neq B$ be an irreducible $U$-submodule of $A$. Assume that $U$ is upper modular in $L$. Then the following hold.

(i) $\langle U, a\rangle \cap A$ is irreducible as a $U$-submodule, for every $0 \neq a \in A$.

(ii) If $B \subseteq N$, then $B$ is an abelian subalgebra of $L$.

(iii) $\langle U, b\rangle=\langle U, B\rangle=U+B$ for every $0 \neq b \in B$.

(iv) $A$ is completely reducible as a $U$-module.

(v) If $U$ is abelian, then the minimum polynomial of $\left.(\operatorname{ad} u)\right|_{A}$ is irreducible, for every $u \in U$.

Proof. (i) First, take $0 \neq n \in N$. Since $N$ is an ideal of $L$, the subspace $N+U$ is a subalgebra of $L$. So $\langle U, n\rangle \subseteq N+U$. This gives $\langle U, n\rangle \cap N=\langle U, n\rangle \cap A$. Also, we have that $\langle U, n\rangle \cap N$ is an ideal of $\langle U, n\rangle$. On the other hand, since $F n \cap U=0$, we have that $U$ is maximal in $\langle U, n\rangle$ because of its upper modularity. It follows that $\langle U, n\rangle \cap N$ is a minimal ideal of $\langle U, n\rangle$. As $N$ is solvable, we find that $\langle U, n\rangle \cap N$ is a solvable minimal ideal of $\langle U, n\rangle$ and so is abelian. This yields that $\langle U, n\rangle \cap A$ is irreducible as a $U$-module. Now, take $a \in A, a \notin N$. Write $A_{a}=\langle U, a\rangle \cap A$. Suppose $A_{a} \cap N \neq 0$. Then there exists $0 \neq n \in A_{a} \cap N$. We have $\langle U, n\rangle \subseteq\langle U, a\rangle$. Since $U$ is maximal in $\langle U, a\rangle$, it follows that $\langle U, a\rangle=\langle U, n\rangle$. Then, by the above, we have that $\langle U, n\rangle \cap A$ is irreducible and $\langle U, n\rangle \cap A=\langle U, n\rangle \cap N$. But then we have $a \in A_{a} \subseteq N$, which is a contradiction. Therefore, $A_{a} \cap N=0$. Since $A_{a}$ is a $U$-submodule of $A$, we have that $0 \neq A_{a} \cong\left(A_{a}+N\right) / N$ is a $U$-submodule of $A / N \cong V$. Irreducibility of $V$ implies that $A_{a} \cong V$ is irreducible as a $U$-submodule, as required.

(ii) Assume that $B \subseteq N$. Pick $0 \neq b \in B$. By Lemma 3.3(i), we have $\langle U, B\rangle=\langle U, b\rangle$. So $B \subseteq\langle U, b\rangle \cap A$. Moreover, in the proof of (i), we have obtained that $\langle U, b\rangle \cap A$ is an abelian subalgebra of $L$.

(iii) If $B \subseteq N$, then $B+U$ is a subalgebra of $L$ because $B$ is abelian (by (ii) above). Assume then that $B \nsubseteq N$. Then we have $N \cap B=0$ by the irreducibility of $B$. Thus $A=$ $N \oplus B$, since $A / N$ is irreducible. Now pick $0 \neq b \in B$. By Lemma 3.3 (i), $\langle U, B\rangle=\langle U, b\rangle$. By (i), $\langle U, b\rangle \cap A$ is irreducible as a $U$-module. This gives $B=\langle U, B\rangle \cap A$. On the other hand, since $L=U \oplus A$, we have $\langle U, B\rangle=U+\langle U, B\rangle \cap A$. Therefore, $\langle U, B\rangle=U+B$.

(iv) Since $A=\sum_{a}(A \cap\langle U, a\rangle)$, it follows from (i) that $A$ is completely reducible as a $U$-module.

(v) Assume that $U$ is abelian. Then we know that the minimum polynomial of $\left.(\operatorname{ad} u)\right|_{B}$ is irreducible, for every irreducible $U$-module $B$ and for every $u \in U$. By (i), we have that every non-zero element of $A$ is contained in an irreducible $U$-submodule of $A$. From this follows (v). 


\section{Solvable Lie algebras}

First we consider the case where the ground field is the real field. In this case, we are able to determine the structure of solvable Lie algebras $L$ having an abelian upper-modular subalgebra $U$ which is not an ideal of $L$ and such that $U \cap L^{2}=0$.

The abelian socle of $L, \operatorname{Asoc}(L)$, is the sum of the minimal abelian ideals of $L$. The Frattini subalgebra of $L, F(L)$, is the intersection of all the maximal subalgebras of $L$, and the Frattini ideal of $L, \phi(L)$, is the largest ideal of $L$ contained in $F(L)$. If $\phi(L)=0$, we say that $L$ is $\phi$-free.

Theorem 4.1. Let $F$ be the real field. Let $L$ be a solvable Lie algebra and let $U$ be an abelian subalgebra which is not an ideal of $L$ and such that $U \cap L^{2}=0$. Then the following are equivalent.

(i) $U$ is upper modular in $L$.

(ii) $L^{2} \subseteq \operatorname{Asoc}(L), L=L^{2} \oplus U$ and the minimum polynomial of $\left.(\operatorname{ad} u)\right|_{L^{2}}$ is irreducible for every $u \in U$.

(iii) $L$ is $\phi$-free, $L^{2}$ is abelian, $U$ is a Cartan subalgebra of $L$ and the minimum polynomial of $\left.(\operatorname{ad} u)\right|_{L^{2}}$ is irreducible for every $u \in U$.

Proof. (i) $\Rightarrow$ (ii). By Lemma 2.1, we have that $U$ is a Cartan subalgebra of $L$. So $L=L^{2} \oplus U$ and $L^{2}$ is just the Fitting one-component of $L$ relative to $U$. By Theorem $3.6(\mathrm{v})$, we have that the minimum polynomial, $m_{u}(x)$, of $\left.(\operatorname{ad} u)\right|_{L^{2}}$ is irreducible for every $u \in U$. It is well known that there exists $u_{0} \in U$ acting non-singularly on $L^{2}$. If $m_{u_{0}}(x)=(x-t)$ for some $t \in F$, then we see that $L^{2}$ is precisely the eigenspace of $L$ corresponding to the eigenvalue $t$ relative to ad $u_{0}$. So, by using the Jacobi identity, we obtain $\left[L^{2}, L^{2}\right]=0$. Now suppose $\operatorname{deg}\left(m_{u_{0}}(x)\right)=2$. Let $\Omega$ be the complex field. Decompose $m_{u_{0}}(x)=(x-\alpha)(x-\beta)$, where $\alpha, \beta \in \Omega$. Let $L_{\Omega}=L \otimes_{F} \Omega$, and decompose $\left(L^{2}\right)_{\Omega}=V_{\alpha} \oplus V_{\beta}$ into its eigenspaces relative to ad $u_{0}$. We have $\left[V_{\alpha}, V_{\beta}\right] \subseteq V_{\alpha+\beta}=0$, since $u$ acts non-singularly on $L^{2}$. Since, clearly, the other products are zero, we have $\left[L^{2}, L^{2}\right]=0$. We conclude that $L^{2}$ is abelian. Then we see that the irreducible $U$ submodules of $L^{2}$ are precisely the minimal ideals of $L$ contained in $L^{2}$. Since, by Theorem 3.6 (iv), $L^{2}$ is completely reducible as a $U$-submodule, it follows that $L^{2} \leqslant \operatorname{Asoc}(L)$.

(ii) $\Rightarrow$ (i). Since $L^{2} \subseteq \operatorname{Asoc}(L)$, it follows that $L^{2}$ is completely reducible as a $U$ submodule of $L$ and that the irreducible $U$-submodules of $L^{2}$ are precisely the minimal ideals of $L$ contained in $L^{2}$. Since $L^{2}$ is abelian and $L=L^{2} \oplus U$, it follows from Proposition 3.5 and Theorem 3.4 that $U$ is upper modular.

(ii) $\Leftrightarrow$ (iii). See Proposition 1 of $[7]$.

Clearly, subalgebras $U$ of dimension one which are not ideals satisfy the conditions in Theorem 4.1. In this case, we are able to give the multiplication table of such Lie algebras.

Corollary 4.2. Let $F$ be the real field. Then a solvable Lie algebra $L$ has a non-ideal minimal subalgebra of $L$ which is upper modular if and only if one of the following holds. 
(i) $L$ has a basis $a_{1}, \ldots, a_{r}, u$ with product given by $\left[u, a_{i}\right]=a_{i}$ for every $i$ and the other products are zero.

(ii) $L$ has a basis $a_{1}, \ldots, a_{r}, b_{1}, \ldots, b_{r}, u$ with product given by $\left[u, a_{i}\right]=b_{i},\left[u, b_{i}\right]=$ $\beta a_{i}+\alpha b_{i}$ for every $i$, where $\alpha, \beta \in F$ and the polynomial $x^{2}-\alpha x-\beta$ is irreducible over $F$.

For any infinite perfect field, we need to impose the condition that $L^{2}$ is abelian.

Theorem 4.3. Let $F$ be any infinite perfect field. Let $L$ be a metabelian Lie algebra. Let $U$ be an abelian subalgebra which is not an ideal of $L$ and such that $L^{2} \cap U=0$. Then the following are equivalent.

(i) $U$ is upper modular.

(ii) $L^{2} \leqslant \operatorname{Asoc}(L), L=L^{2} \oplus U$ and the minimum polynomial of $\left.(\operatorname{ad} u)\right|_{L^{2}}$ is irreducible for every $u \in U$.

(iii) $L$ is $\phi$-free, $U$ is a Cartan subalgebra of $L$ and the minimum polynomial of $\left.(\operatorname{ad} u)\right|_{L^{2}}$ is irreducible for every $u \in U$.

Proof. (i) $\Rightarrow$ (ii). Since $U+L^{2}$ is an ideal of $L$ properly containing $U$, Theorem 2.2 implies that $L=L^{2} \oplus U$. From Theorem 3.6 (iv), it follows that $L^{2}$ is completely reducible as a $U$-submodule of $L$. On the other hand, since $L^{2}$ is abelian, we see that the irreducible $U$-submodules of $L^{2}$ are precisely the minimal ideals of $L$ contained in $L^{2}$. This yields $L^{2} \subseteq \operatorname{Asoc}(L)$. Moreover, by Theorem $3.6(\mathrm{v})$, it follows that the minimum polynomial of $\left.(\operatorname{ad} u)\right|_{L^{2}}$ is irreducible for every $u \in U$. The proof of the remaining parts are as in Theorem 4.1.

For arbitrary fields, we have the following result.

Theorem 4.4. Let $L$ be a solvable Lie algebra over any field $F$, and let $U$ be an abelian subalgebra of $L$ which is not an ideal of $L$ and such that $U \cap L^{2}=0$. Let $U$ be upper modular. Then, $L=L^{2} \oplus U, L^{2}$ is completely reducible as a $U$-submodule of $L$, every irreducible $U$-submodule of $L^{2}$ is an abelian subalgebra of $L$ and the minimum polynomial of $\left.(\operatorname{ad} u)\right|_{L^{2}}$ is irreducible for every $u \in U$.

Proof. This follows from Theorem 3.6.

For minimal subalgebras, the converse of Theorem 4.4 holds.

Proposition 4.5. Let $L$ be a solvable Lie algebra over any field $F$ and let $u \in L$. Then $F u$ is upper modular in $L$ if and only if either of the following hold.

(i) $F u$ is an ideal of $L$.

(ii) $L=L^{2} \oplus F u$, every subspace of $L^{2}$ that is invariant and irreducible under ad $u$ is an abelian subalgebra of $L$ and the minimum polynomial of $\left.(\mathrm{ad} u)\right|_{L^{2}}$ is irreducible. 
Proof. Suppose that (i) or (ii) holds; ideals are clearly upper modular, so suppose (ii) holds. Then $\left.(\operatorname{ad} u)\right|_{L^{2}}$ is semisimple, so $L^{2}$ is completely reducible as an $F u$-module. Also, from the hypothesis that the minimum polynomial of $\left.(\operatorname{ad} u)\right|_{L^{2}}$ is irreducible, it follows that each element in $L^{2}$ lies in an irreducible $F u$-submodule of $L^{2}$. The fact that $F u$ is upper modular in $L$ is now immediate from Theorem 3.4. The converse follows from Theorem 4.4 .

\section{Non-solvable Lie algebras}

For any field of characteristic zero, we are able to classify the non-solvable Lie algebras having an upper-modular minimal subalgebra which is not an ideal. There exist such Lie algebras if and only if $\sqrt{F} \nsubseteq F$.

If $u \in L$, we shall write $C_{L}(u)$ for $\{x \in L:[x, u]=0\}$.

Theorem 5.1. Let $L$ be a non-solvable Lie algebra over a field $F$ of characteristic zero and let $u \in L$. Then $F u$ is an upper-modular subalgebra of $L$ if and only if one of the following holds.

(i) $F u$ is an ideal of $L$.

(ii) The solvable radical $R(L)$ of $L$ is abelian, $u$ lies in a Levi subalgebra $S$ of $L$ which is three-dimensional simple with basis $u, v, w$ such that $[u, v]=w,[v, w]=4 \alpha u$, $[w, u]=4 \beta v$, where $\alpha, \beta \in F$ and $\sqrt{-\beta} \notin F, L=R(L) \oplus S$ and the minimum polynomial of $\left.(\operatorname{ad} u)\right|_{R(L)+F v+F w}$ is $\left(x^{2}+4 \beta\right)$.

Proof. Let $F u$ be an upper-modular subalgebra of $L$ which is not an ideal of $L$. By Levi's theorem, we can write $L=R \oplus S$, where $R$ is a maximal ideal of $L$ containing the radical of $L$ and $S$ is a simple subalgebra of $L$. Suppose that $u \notin S$. Then $u=r+s$, where $0 \neq r \in R, s \in S$. Now there is an element $t \in S$ such that $\langle s, t\rangle=S$ (see, for instance, Theorem 5 of $[8])$. Put $T=\langle u, t\rangle$. Then

$$
R+T=R+\langle u, t\rangle=R+\langle s, t\rangle=L,
$$

so $T /(T \cap R) \cong L / R \cong S$ is simple.

Suppose that $u \in T \cap R$. Since $T \cap R$ is an ideal of $T$, it follows from Theorem 2.2 that $F u$ is an ideal of $T$. But then $N_{L}(F u) \neq F u$, and so $F u$ is an ideal of $L$, by Lemma 2.1, contrary to our assumption. Hence $u \notin T \cap R$.

Suppose now that $T \cap R \neq 0$. Then $T=(T \cap R) \oplus F u$, since $F u$ is a maximal subalgebra of $T$. But this implies that $T /(T \cap R)$ is one dimensional, contradicting the fact that it is simple. This yields $T \cap R=0$ and $u \in T \cong S$, which is simple. Replacing $S$ by $T$ if necessary, we may assume that $u \in S$.

But $F u$ is upper modular in $S$, and hence a maximal subalgebra of $S$, as above. It follows that $S$ is three-dimensional non-split simple. Moreover, if $\Sigma$ is a Levi factor of $L$ containing $S$, then $F u$ is a maximal subalgebra of $\Sigma$, and so $\Sigma=S$ and $R=R(L)$ is the radical of $L$. Therefore, $L=R(L) \oplus S$. Also, the element $u$ can be complemented by elements $v, w$ to form a basis for $S$ with products given by $[u, v]=w,[v, w]=4 \alpha u$, 
$[w, u]=4 \beta v$, where $\alpha, \beta \in F$ and $\sqrt{-\beta} \notin F$. Write $V=F v+F w$. We see that $V$ is an irreducible $F u$-submodule of $L$ and that the minimum polynomial of $\left.(\operatorname{ad} u)\right|_{V}$ is $x^{2}+4 \beta$. Write $M=R(L) \oplus V$. If $R(L)=0$, then case (ii) holds; so suppose that $R(L) \neq 0$. By Theorem 3.6, it follows that $R(L)$ is completely reducible as an $F u$-module and the minimum polynomial of $\left.(\operatorname{ad} u)\right|_{M}$ is irreducible. This gives that the minimum polynomial of $\left.(\operatorname{ad} u)\right|_{R(L)}$ is also $x^{2}+4 \beta$. Moreover, we see that the adjoint action of $u$ on $R(L)$ is non-singular.

Let $\Omega$ be an algebraic closure of $F$ and let $R(L)_{\Omega}=R(L) \otimes_{F} \Omega$. We can decompose $x^{2}+4 \beta=(x-\omega)(x+\omega)$, where $\omega \in \Omega$. This implies that we can decompose $R(L)_{\Omega}=\left(R(L)_{\Omega}\right)_{\omega} \oplus\left(R(L)_{\Omega}\right)_{-\omega}$ into its root spaces relative to ad $u$. We find $\left[\left(R(L)_{\Omega}\right)_{\omega},\left(R(L)_{\Omega}\right)_{-\omega}\right] \subseteq\left(R(L)_{\Omega}\right)_{0}=0$, and the other products are also equal to zero. This yields that $R(L)$ is abelian. So, we have case (ii).

It remains to show that, in case (ii), $F u$ must be upper modular in $L$. We write $V=$ $F v+F w$. In this case, $\left.(\operatorname{ad} u)\right|_{R(L)+F v+F w}$ is semisimple, and so $R(L)+V$ is completely reducible as an $F u$-module. Also, we have that, for every non-zero element in $R(L)+V$, the cyclic $F u$-module generated by it is irreducible as an $F u$-module. On the other hand, we see $V+F u=S$, and so $V+F u$ is a subalgebra of $L$. Now let $B$ be any irreducible $F u$-submodule of $L$ contained in $R(L) \oplus V$. If $B \subseteq R(L)$, then we have that $B+F u$ is a subalgebra of $L$, since $R(L)$ is abelian. Now assume $B \nsubseteq R(L)$. Then $B \cap R(L)=0$ and $R(L) \oplus B$ is a $F u$-submodule of $R(L) \oplus V$. Since the latter is a completely reducible $F u$-submodule, there exists a complement $C$ such that $R(L) \oplus B \oplus C=R(L) \oplus V$. Consequently, $\operatorname{dim} B \leqslant 2$. If $\operatorname{dim} B=1$, then $B$ is abelian and $B+F u$ is a subalgebra of $L$. Hence only the case $\operatorname{dim} B=2$ remains to be considered. Let $\Omega$ be an algebraic closure of $F$ and let $B_{\Omega}=B \otimes_{F} \Omega$. Decompose, as above, $x^{2}+4 \beta=(x-\omega)(x+\omega)$, where $\omega \in \Omega$. Let $B_{\Omega}=\left(B_{\Omega}\right)_{\omega} \oplus\left(B_{\Omega}\right)_{-\omega}$ be the decomposition of $B_{\Omega}$ into its root spaces relative to ad $u$. Now we have that $\left[u,\left[\left(B_{\Omega}\right)_{\omega},\left(B_{\Omega}\right)_{-\omega}\right]\right]=0$. This yields $[B, B] \subseteq C_{L}(u)$. On the other hand, as $x^{2}+4 \beta$ is the minimum polynomial of $\left.(\operatorname{ad} u)\right|_{R(L)+B}$, we see that $C_{L}(u) \cap(R(L)+B)=0$. This yields $C_{L}(u)=F u$, and therefore $[B, B] \subseteq F u$. It follows that $B+F u$ is a subalgebra of $L$. Then, by Theorem 3.4, it follows that $F u$ is upper modular in $L$. Now the proof is complete.

Corollary 5.2. Let $F$ be any field of characteristic zero. Let $L$ be a non-solvable Lie algebra. Then $L$ has an upper-modular minimal subalgebra which is not an ideal if and only if $L$ has a basis $a_{1}, \ldots, a_{r}, b_{1}, \ldots, b_{r}, u, v, w$ with product given by $\left[u, a_{i}\right]=b_{i}$, $\left[u, b_{i}\right]=-4 \beta a_{i},[u, v]=w,[v, w]=4 \alpha u,[w, u]=4 \beta v$ and the other products zero, where $\alpha, \beta \in F$ and $\sqrt{-\beta} \notin F$.

Corollary 5.3. Over fields $F$ of characteristic zero such that $\sqrt{F} \subseteq F$, a Lie algebra $L$ having an upper-modular minimal subalgebra which is not an ideal of $L$ must be solvable.

\section{6. $\operatorname{um}(0)$-algebras}

We shall call $L$ a um(0)-algebra if all of its one-dimensional subalgebras are upper modular in $L$. The Lie algebra $L$ is almost abelian if $L=L^{2} \oplus F x$, where $L^{2}$ is abelian 
and $[x, y]=y$ for all $y \in L^{2}$; if $L$ is abelian or almost abelian, we say that it is quasiabelian. In [2], it was shown that, over a field of characteristic zero, $L$ is a um(0)-algebra if and only if it is quasi-abelian or three-dimensional non-split simple. Here we want to generalize this result by considering a wider class of fields.

A $\mu$-algebra is a non-solvable Lie algebra in which every non-zero proper subalgebra is one dimensional. Every $\mu$-algebra $L$ is supersimple; that is, every subalgebra of $L$ of dimension greater than one is simple. Over a perfect field of characteristic different from two and three, the only supersimple Lie algebras are the three-dimensional non-split simple Lie algebras (see Proposition 1 of [4]). The generalization we obtain is that, over a field of characteristic different from two and three, $L$ is a um(0)-algebra if and only if it is quasi-abelian or a $\mu$-algebra. First we need the following result, which is related to Lemma 4.1 of $[\mathbf{3}]$.

Theorem 6.1. Let $L$ be a Lie algebra over any field $F$ and suppose that every twogenerated subalgebra of $L$ is either quasi-abelian or a $\mu$-algebra. Then either of the following hold.

(i) $L$ is quasi-abelian.

(ii) Every two-generated subalgebra of $L$ is a $\mu$-algebra.

Proof. Suppose first that every two-generated subalgebra of $L$ is quasi-abelian. Then every two-dimensional subspace of $L$ is a subalgebra of $L$, from which it is clear that every subspace of $L$ is a subalgebra of $L$. It follows that $L$ is quasi-abelian.

So suppose that $L$ has two-generated subalgebras $Q, U$, where $Q$ is quasi-abelian and $U$ is a $\mu$-algebra. We claim that we may assume that $Q=\langle x, y\rangle$ and $U=\langle y, z\rangle$ for some $x, y, z \in L$. Clearly, either $Q \cap U=0$ or $Q \cap U=F a$ for some $0 \neq a \in L$. Suppose first that $Q \cap U=0$. Choose any $0 \neq q \in Q, 0 \neq u \in U$. Then $\langle q, u\rangle$ is either quasi-abelian or a $\mu$-algebra. If it is quasi-abelian, take $x=q, y=u$ and $z \in U \backslash\langle q, u\rangle$; if it is a $\mu$-algebra, take $x \in Q \backslash\langle q, u\rangle, y=q$ and $z=u$. Now suppose that $Q \cap U=F a$. Then take $x \in Q \backslash F a, y=a$ and $z \in U \backslash F a$. This establishes our claim.

Suppose next that $Q \cap U=F y$ is an ideal of $Q$, so that $[x, y]=\alpha y$ for some $\alpha \in F$. Then $[y, z] \notin F y$, whence $F y \subset\langle y,[y, z]\rangle \subseteq\langle y, z\rangle$, giving $\langle y,[y, z]\rangle=\langle y, z\rangle$. However, $[x+z, y]=\alpha y+[z, y]$, so $\langle y, z\rangle=\langle y,[y, z]\rangle \subseteq\langle x+z, y\rangle$. But now $\langle x+z, y\rangle$ is a twogenerated subalgebra of $L$ containing both $Q$ and $U$, which is impossible.

It follows that $Q \cap U=F y$ is not an ideal of $Q$. There is an element $q \in Q$ for which $F q$ is an ideal of $Q$, and, without loss of generality, we may assume that $q=x$. Thus $[x, y]=\beta x$ for some $\beta \in F$. Then $[x+z, y]=\beta x+[z, y] \in\langle x+z, y\rangle$, so

$$
-\beta z-[y, z]=-\beta z+[z, y]=-\beta(z+x)+\beta x+[z, y] \in\langle x+z, y\rangle .
$$

Hence $\beta z+[y, z] \in\langle x+z, y\rangle$. Also, $\beta z+[y, z] \notin F y$, since $\langle y, z\rangle$ is a $\mu$-algebra, from which it follows that $\langle y, z\rangle=\langle\beta z+[y, z], y\rangle \subseteq\langle x+z, y\rangle$. This means that $\langle x+z, y\rangle$ is a $\mu$-algebra, because otherwise $U=\langle y, z\rangle$ would be solvable. It follows that $\langle y, z\rangle=\langle x+z, y\rangle$ and $x \in\langle x, y\rangle \cap\langle y, z\rangle=F y$, which is a contradiction. The result now follows. 
Lemma 6.2. Let $L$ be a Lie algebra over any field $F$. Then the following are equivalent.

(i) $L$ is supersimple.

(ii) Every subalgebra of $L$ is either one dimensional or simple.

(iii) $L$ has no two-dimensional subalgebras.

Proof. The equivalence of (ii) and (iii) is given by Varea in [11]. Since two-dimensional subalgebras are never simple, (i) implies (iii). Finally, it is trivial that (ii) implies (i).

We shall need the following property of supersimple Lie algebras which was given by Varea in $[\mathbf{1 2}]$.

Theorem 6.3. Let $L$ be a supersimple Lie algebra over a field $F$ of characteristic $p$ different from 2, 3. Then, for each $0 \neq x \in L$, there is a $y \in L$ such that $\langle x, y\rangle=L$.

Proof. This is Theorem 4 of [12].

The above result has the following consequence for um(0)-algebras.

Lemma 6.4. Let $L$ be a supersimple um(0)-algebra over a field of characteristic different from 2,3 . Then $L$ is a $\mu$-algebra.

Proof. Choose any $0 \neq x \in L$. Then, by the above theorem, there is a $y \in L$ such that $\langle x, y\rangle=L$. Now $F x \cap F y=0$, which is a maximal subalgebra of $F y$, so $F x$ is a maximal subalgebra of $L$. It follows that every one-dimensional subalgebra of $L$ is maximal in $L$, and hence that $L$ is a $\mu$-algebra.

We can now prove our main result.

Theorem 6.5. Let $L$ be any Lie algebra over a field $F$ of characteristic different from 2, 3. Then the following are equivalent.

(i) $L$ is a um(0)-algebra.

(ii) $L$ is quasi-abelian or a $\mu$-algebra.

Proof. Suppose first that $L$ is a um(0)-algebra, and let $U=\langle x, y\rangle$ be a two-generated subalgebra of $L$ which is not quasi-abelian. Then $U$ has dimension greater than two, and $F x, F y$ are maximal subalgebras of $U$. Suppose that $U$ has a two-dimensional subalgebra, $S=F a+F b$, say. Clearly, we can assume that $x \notin S$, so that $\langle x, s\rangle=U$ for every $0 \neq s \in S$. But now $F x \cap F a=0$, which is a maximal subalgebra of $F x$, so $F a$ is maximal in $\langle x, a\rangle=U$. This contradicts the fact that $F a \subset S \subset U$. It follows that $U$ has no two-dimensional subalgebras, and so is supersimple, by Lemma 6.2. But now $U$ must be a $\mu$-algebra, by Lemma 6.4. We have shown that every two-generated subalgebra of $L$ is either quasi-abelian or a $\mu$-algebra. We conclude from Theorem 6.1 that either $L$ is quasi-abelian or else every two-generated subalgebra of $L$ is a $\mu$-algebra. In the latter case, $L$ is supersimple, by Lemma 6.2 , and hence a $\mu$-algebra, by Lemma 6.4.

The converse is easy to check. 
Acknowledgements. V.R.V. was supported by DGI grant BFM2000-1049-C02-01. The authors are grateful to the referee for his/her suggestions.

\section{References}

1. D. W. BARnes, On Cartan subalgebras of Lie algebras, Math. Z. 101 (1967), 350-355.

2. K. Bowman AND D. A. Towers, Modularity conditions in Lie algebras, Hiroshima Math. J. 19 (1989), 333-346.

3. K. Bowman and V. R. Varea, Modularity* in Lie algebras, Proc. Edinb. Math. Soc. 40 (1997), 99-110.

4. A. G. GEIN, Modular rule and relative complements in the lattice of subalgebras of a Lie algebra, Sov. Math. Dokl. 31(3) (1987), 22-32 (translated from Izv. Vyssh. Uchebn. Zaved. Mat. 83 (1987), 18-25).

5. A. G. GEIN AND V. R. VAREA, Solvable Lie algebras and their subalgebra lattices, corrigenda, Commun. Alg. 23(1) (1992), 399-403.

6. N. Jacobson, Lie algebras (Dover, New York, 1979).

7. E. L. Stitzinger, Frattini subalgebras of a class of solvable Lie algebras, Pac. J. Math. 34 (1970), 177-182.

8. D. A. Towers, On complemented Lie algebras, J. Lond. Math. Soc. 22 (1980), 63-65.

9. D. A. Towers, Lattice isomorphisms of Lie algebras, Math. Proc. Camb. Phil. Soc. 89 (1981), 285-292.

10. D. A. Towers, Semimodular subalgebras of a Lie algebra, J. Alg. 103 (1986), 202-207.

11. V. R. VAREA, Lie algebras none of whose Engel subalgebras are in intermediate position, Commun. Alg. 15(12) (1987), 2529-2543.

12. V. R. VAreA, Supersimple and upper semimodular Lie algebras, Commun. Alg. 23(6) (1995), 2323-2330. 